\title{
Factores de riesgo asociados a lesiones precancerosas del cuello uterino en pacientes atendidas en consultorio de ginecología Hospital Víctor Ramos Guardia Huaraz, 2014-2015
}

Risk factors associated with precancerous lesions of the cervix in women seen in the gynecology clinic Hospital Víctor Ramos Guardia Huaraz, 2014-2015

\author{
Maximiliana Quispe Gómez ${ }^{1}$, Consuelo Valencia Vera ${ }^{1}$, Ada Rodríguez Figueroa ${ }^{1}$ Y \\ Patricia Salazar Quispe ${ }^{2}$
}

\section{RESUMEN}

La investigación tuvo como objetivo determinar los factores de riesgo asociados a lesiones precancerosas del cuello uterino en pacientes atendidas en el Hospital Víctor Ramos Guardia-Huaraz, 2014-2015. Investigación observacional, analítica, de Casos y Controles, con una muestra de 183 mujeres con lesiones cervicales(Casos) y 183 mujeres sin lesiones cervicales (Controles). Se usó como instrumento una ficha de recolección de datos validada por la institución de estudio, la información se procesó mediante el programa SPSS V20, realizándose la contrastación de la hipótesis mediante las pruebas estadísticas el $\mathrm{chi}^{2}$ y Odss Ratio (OR). Los resultados obtenidos fueron: Edad, mayores de 35 años $(\mathrm{OR}=1,94)$, bajo grado de instrucción $(\mathrm{OR}=1,84)$, estado civil de convivencia $(\mathrm{OR}=1,67)$, procedencia rural $(\mathrm{OR}=3,92)$, inicio de las relaciones sexuales $<$ de 16 años $(\mathrm{OR}=29,51)$, más de 2 parejas sexuales $(\mathrm{OR}=16,06)$, Papiloma Virus Humano $(\mathrm{VPH})(\mathrm{OR}=2,51)$, multiparidad $(\mathrm{OR}=17,21)$, uso de métodos anticonceptivos hormonales y antecedentes familiares $(\mathrm{OR}=3,13)$. Ante lo cual se concluyó que los factores de riesgo personal, sexual y reproductivo mostraron asociación significativa con las lesiones precancerosas del cuello uterino.

Palabras clave: factores de riesgo; lesiones precancerosas del cuello uterino; lesiones cervicales.

\footnotetext{
1 Universidad Nacional Santiago Antúnez de Mayolo. Huaraz, Perú.

2 Universidad de Aquino Bolivia. Cochabamba, Perú.
}

(C) Los autores. Este artículo es publicado por la Revista Aporte Santiaguino de la Universidad Nacional Santiago Antúnez de Mayolo. Este es un artículo de acceso abierto, distribuido bajo los términos de la Licencia Creative Commons Atribución-NoComercial-CompartirIgual 4.0 Internacional. (http://creativecommons.org/licenses/ by-nc-sa/4.0/), que permite el uso no comercial, distribución y reproducción en cualquier medio, siempre que la obra original sea debidamente citada. 


\section{ABSTRACT}

The objective research was to determine the risk factors associated with precancerous lesions of the cervix in patients treated in the Victor Ramos Guardia-Huaraz Hospital, 2014-2015. Observational and analytical research of cases and controls, with a sample of 183 women with cervical lesions (Cases) and 183 women without cervical lesions (Control group). A data collection form validated by the hospital was used as an instrument; the information was processed through the SPSS V20 program, contrasting the hypothesis through the Chi Square test and Odss Ratio (OR) statistical tests. The obtained results were: Their age, over $35(\mathrm{OR}=1,94)$, with low educational level $(\mathrm{OR}=1,84)$, with a cohabiting marital status $(\mathrm{OR}=1,67)$, from rural origin $(\mathrm{OR}=3,92)$, 16 , as the age of starting having sex $(\mathrm{OR}=29,51)$, more than 2 sexual partners (OR =16,06), suffered from Human Papilloma Virus (HPV) (OR = 2,51), were multiparous $(\mathrm{OR}=17,21)$, used hormonal contraceptive methods and had family history $(\mathrm{OR}=$ $3,13)$. So, the conclusion was that the personal, sexual and reproductive risk factors showed significant association with the precancerous lesions of the cervix.

Keywords: risk factors; precancerous lesions of the cervix; cervical lesions.

\section{INTRODUCCIÓN}

En los últimos años el Cáncer de Cuello Uterino (CACU) se ha convertido en un problema de salud pública a nivel mundial y en nuestro país, específicamente referente a la identificación de las lesiones precancerosas y los factores de riesgo, se considera como una de las causas más frecuentes de muerte en las mujeres de los países en desarrollo y sus tasas de incidencias más altas se encuentran en Centro y Sur de América, África Subsahariana, sureste de Asia y el Caribe. Anualmente se registran aproximadamente 500,000 casos, de esos, 230,000 fallecen, el 80\% ocurre en países en desarrollo. Más de 300,000 mujeres mueren anualmente y de ellas más del 70\% en plena capacidad reproductiva. En Europa, el CACU es la tercera causa de muerte en las mujeres y en Latinoamérica, es el cuarto tipo de cáncer más frecuente, se estima que cada año se diagnostican 83,200 casos nuevos y que 35,680 mujeres mueren por este tipo de cáncer. En el Perú, el Cáncer de Cuello Uterino es la primera causa de muerte por cáncer en las mujeres, y la segunda en Lima después del cáncer de mama. La Organización Panamericana de la Salud (OPS), ha declarado que el CACU, es una enfermedad que se puede prevenir en gran medida, sea por medio de la vacunación contra el Virus del Papiloma Humano (VPH) en los primeros años de la adolescencia o bien mediante las 
pruebas de detección y el tratamiento de lesiones precancerosas en mujeres de 30 años en adelante, inicialmente estas lesiones presentan cambios en las células que cubren las paredes del cuello uterino y evolucionan a cáncer si no son diagnosticadas y tratadas oportunamente. Se hace mención de que el factor de riesgo más común es la exposición a ciertas variedades del VPH. Siendo esta una Infección de Transmisión Sexual (ITS) en muchos casos asintomáticos, por lo que puede transcurrir mucho tiempo antes de que se detecte. A menudo las mujeres se infectan con el VPH en edades entre los 20 y 30 años, solo una minoría desarrolla el cáncer, proceso que puede tomar hasta 20 años. El CACU, tiene importancia médica, socioeconómica y humana; es el más curable, más fácil de diagnosticar, el más prevenible de todos los cánceres y es el principal problema de salud en las mujeres en actividad sexual de los países subdesarrollados por no tener en forma deficiente programas de detección temprana y existen múltiples factores asociados a la falta de tamizaje.

Hay muchos trabajos de investigación sobre los factores de riesgo que influyen en el desarrollo de las lesiones precancerosas del cuello uterino, concluyen que existen factores de riesgo personal, social, y sexual; de los cuales los factores de riesgo más significativos fueron: el número de parejas sexuales, antecedentes heredofamiliares, infección del VPH, las relaciones sexuales a temprana edad (antes de 16 años), la promiscuidad, el número de partos, el riesgo aumenta a mayor número de partos, abortos a temprana edad; factores exógenos como el tabaquismo, el VIH u otras ITS como el herpes simple tipo 2.

En la Región de Áncash según los datos obtenidos de la Dirección Regional de Salud Áncash (DIRESA), en el año 2012, se presentaron 185 casos de displasia cervical leve, 97 casos de displasia cervical severa, 14 casos de displasia cervical moderada y 13 casos de displasia del cuello del útero no especificada durante el periodo 2014-2015, en el cual se notificó 198 casos de enfermedad precancerosa del cuello uterino identificadas mediante la toma de Papanicolaou y se detectó 20 casos de CACU. Motivo por el cual se realizó la investigación con el objetivo general de determinar los factores de riesgo asociados con lesiones precancerosas del cuello uterino en pacientes atendidas en el consultorio de ginecología del Hospital Víctor Ramos Guardia-Huaraz, 2014-2015, con los objetivos específicos de identificar si los factores personales se asocian a lesiones precancerosas en el cuello uterino; determinar si los factores sexuales se asocian a lesiones precancerosa en el cuello uterino; identificar si los factores reproductivos se asocian a lesiones precancerosas en el cuello uterino; y finalmente determinar el tipo de lesión precancerosa más frecuente del cuello uterino. 


\section{MATERIALES Y MÉTODOS}

En concordancia con el problema planteado y los objetivos de la investigación se realizó un estudio epidemiológico analítico de Casos y Controles (Bonita, 2008). La población de estudio estuvo conformada por las historias clínicas de mujeres que se tomaron el Papanicolaou (PAP) en el consultorio de ginecología del Hospital Víctor Ramos Guardia-Huaraz-Áncash, en el periodo 2014- 2015, con una muestra (casos) 183 historias clínicas de pacientes con resultados de PAP positivos a lesiones precancerosas de cuello uterino y (controles)183 historias clínicas de pacientes con resultado de PAP negativo. Como instrumento de recolección de datos, se usó una ficha de recolección de información previamente diseñado en base al formato institucional de "Solicitud y Reporte de Resultados de Citología Cervical” del Programa de Prevención y Control de Cáncer Cérvico Uterino y validada por la institución de estudio. Dicho instrumento abarcó las diversas variables y los diversos factores presentados en la investigación. Los resultados del PAP como instrumento de recolección de datos reúnen los requisitos indispensables de confiabilidad, validez y objetividad; para el procesamiento y análisis de la información se usó el programa estadístico SPSS V20 y la distribución de tablas, así como la prueba estadística chi $^{2}$ y para la medida de fuerza de asociación se utilizó la razón de oportunidades o razón de probabilidades Odds Ratio (OR) con intervalo de confianza del 95\%.

En la investigación se emplearon como fuentes secundarias, las historias clínicas, resultados de los formatos de PAP que se obtuvieron en el Hospital Víctor Ramos Guardia-Huaraz en el período de estudio para lograr los objetivos y fines propuestos. Los datos que se han obtenido del instrumento de recolección de datos, se han utilizado solo con fines de investigación y existe el compromiso de confidencialidad y privacidad de parte de las autoras del presente estudio. 


\section{RESULTADOS}

Tabla 1. Factores de riesgo personales asociados a lesiones precancerosas del cuello uterinario

\begin{tabular}{|c|c|c|c|c|c|c|c|}
\hline \multirow{2}{*}{$\begin{array}{l}\text { Factores } \\
\text { personales }\end{array}$} & \multicolumn{2}{|c|}{$\begin{array}{l}\text { Con lesiones y/o } \\
\text { cáncer cervical }\end{array}$} & \multicolumn{2}{|c|}{$\begin{array}{l}\text { Sin lesiones ni } \\
\text { cáncer cervical }\end{array}$} & \multicolumn{2}{|c|}{ Total } & \multirow{2}{*}{$\begin{array}{l}\text { Resultado análi- } \\
\text { sis estadístico }\end{array}$} \\
\hline & $\mathrm{n}$ & $\%$ & $\mathrm{n}$ & $\%$ & $\mathrm{n}$ & $\%$ & \\
\hline \multicolumn{8}{|l|}{ Edad } \\
\hline $\begin{array}{l}15 \text { a } 24 \\
\text { años }\end{array}$ & 22 & 12,0 & 64 & 35,0 & 86 & 23,5 & $X^{2}=65,159$ \\
\hline 25 a 34 años & 61 & 33,3 & 90 & 49,2 & 151 & $4, .3$ & P-valor $=0,000$ \\
\hline $\begin{array}{l}>\text { de } 35 \\
\text { años }\end{array}$ & 100 & 54,6 & 29 & 15,8 & 129 & 35,2 & $\begin{array}{c}\mathrm{OR}=1,94 \\
\text { IC95\%:1,27-2,95 }\end{array}$ \\
\hline Total & 183 & 100,0 & 183 & 100,0 & 366 & 100,0 & $\begin{array}{c}\text { Exp: }>35 \text { años } \\
\text { No Exp:15-24 } \\
\text { años }\end{array}$ \\
\hline \multicolumn{8}{|l|}{ Estado civil } \\
\hline Soltera & 17 & 9,3 & 61 & 33,3 & 78 & 21,3 & $\mathrm{X}^{2}=57,710$ \\
\hline Conviviente & 107 & 58,5 & 41 & 22,4 & 148 & 40,4 & $P$-valor $=0,00$ \\
\hline Casada & 59 & 32,2 & 81 & 44,3 & 140 & 38,3 & $\mathrm{OR}=1,67$ \\
\hline Total & 183 & 100,0 & 183 & 100,0 & 366 & 100,00 & $\begin{array}{l}\text { IC95\%:1,09-2,56 } \\
\text { Exp: Soltera/conv. } \\
\text { No Exp: Casada }\end{array}$ \\
\hline \multicolumn{8}{|l|}{ Procedencia } \\
\hline Urbana & 115 & 62,8 & 159 & 86,9 & 274 & 74,9 & $X^{2}=28,109$ \\
\hline Rural & 68 & 37,2 & 24 & 13,1 & 25,1 & 25,1 & P-valor $=0,000$ \\
\hline Total & 183 & 100,0 & 183 & 100,0 & 100,0 & 100,0 & $\begin{array}{c}\text { OR=3,92 } \\
\text { IC95\%:2,32-6,61 } \\
\text { Exp.: Rural } \\
\text { No Exp: Urbana }\end{array}$ \\
\hline
\end{tabular}




\begin{tabular}{|c|c|c|c|c|c|c|c|}
\hline \multicolumn{8}{|c|}{ Grado de instrucción } \\
\hline Ninguno & 15 & 8,2 & 7 & 3,8 & 22 & 6,0 & $X^{2}=27,530$ \\
\hline Primaria & 32 & 17,5 & 22 & 12,0 & 54 & 14,8 & P-valor $=0,000$ \\
\hline Secundaria & 97 & 53,0 & 68 & 37,2 & 165 & 45,1 & $\mathrm{OR}=1,84$ \\
\hline Superior & 39 & 21,3 & 86 & 47,0 & 125 & 34,2 & IC95\%:1,09-3,08 \\
\hline \multirow[t]{2}{*}{ Total } & \multirow[t]{2}{*}{183} & \multirow[t]{2}{*}{100,0} & \multirow[t]{2}{*}{183} & \multirow[t]{2}{*}{100,0} & \multirow[t]{2}{*}{366} & \multirow[t]{2}{*}{100,0} & $\begin{array}{c}\text { Exp:.Ning/pri- } \\
\text { maria }\end{array}$ \\
\hline & & & & & & & No Exp: Sec./sup \\
\hline \multicolumn{8}{|c|}{ Antecedente familiar CACU } \\
\hline Hermana & 3 & 1,6 & 3 & 1,6 & 6 & 1,6 & $X^{2}=15,753$ \\
\hline Abuela & 28 & 15,3 & 7 & 3,8 & 35 & 9,6 & P-valor $=0,001$ \\
\hline Madre & 9 & 4,9 & 5 & 2,7 & 14 & 3,8 & $\mathrm{OR}=3,13$ \\
\hline Ninguno & 143 & 78,1 & 168 & 91,8 & 311 & 85,0 & IC95\%:1,66-5,91 \\
\hline Total & 183 & 100,0 & 183 & 100,0 & 366 & 100,0 & $\begin{array}{l}\text { Exp.: Antec. Fam. } \\
\text { No Exp: Ninguno }\end{array}$ \\
\hline
\end{tabular}

Realizado el análisis estadístico se encontró una asociación significativa de los factores de riesgo personales como procedencia rural y antecedentes familiares con las lesiones precancerosas del cuello uterino, con un $\mathrm{OR}=3,92$ y $\mathrm{OR}=3,13$, respectivamente, un intervalo de confianza que no contiene la unidad y un $\mathrm{p}<0,05$; se concluye: La procedencia del área rural y los antecedentes patológicos familiares de CACU constituyeron en factores de riesgo para desarrollar lesiones precancerosas del cuello uterino, el resto de los factores constituyen factores de riesgo aunque no de forma significativa con las lesiones precancerosas del cuello uterino.

Tabla 2. Factores sexuales asociados a lesiones precancerosas del cuello uterino

\begin{tabular}{|c|c|c|c|c|c|c|c|}
\hline \multirow{2}{*}{$\begin{array}{l}\text { Factores } \\
\text { sexuales }\end{array}$} & \multicolumn{2}{|c|}{$\begin{array}{l}\text { Con lesiones y/o } \\
\text { cáncer cervical }\end{array}$} & \multicolumn{2}{|c|}{$\begin{array}{l}\text { Sin lesiones ni } \\
\text { cáncer cervical }\end{array}$} & \multicolumn{2}{|c|}{ Total } & \multirow{2}{*}{$\begin{array}{l}\text { Resultado análi- } \\
\text { sis estadístico }\end{array}$} \\
\hline & $\mathrm{n}$ & $\%$ & $\mathrm{n}$ & $\%$ & $\mathrm{n}$ & $\%$ & \\
\hline \multicolumn{8}{|l|}{ Menarquia } \\
\hline 9 a 10 años & 8 & 4,4 & 3 & 1,6 & 11 & 3,0 & $X^{2}=16,402$ \\
\hline 11 a 12 años & 84 & 45,9 & 61 & 33,3 & 145 & 39,6 & P-valor $=0,001$ \\
\hline 13 a 14 años & 73 & 39,9 & 110 & 60,1 & 183 & 50,0 & $\mathrm{OR}=1,88$ \\
\hline 15 a 16 años & 18 & 9,8 & 9 & 4,9 & 27 & 7,4 & $\begin{array}{c}\text { IC95\%:1,24- } \\
2,86\end{array}$ \\
\hline
\end{tabular}




\begin{tabular}{|c|c|c|c|c|c|c|c|}
\hline Total & 183 & 100,0 & 183 & 100,0 & 366 & 100,0 & $\begin{array}{c}\text { Exp.: M. 9-12 } \\
\text { año } \\
\text { No Exp: M.13- } \\
14 \\
\end{array}$ \\
\hline \multicolumn{8}{|c|}{ Edad de la primera relación sexual } \\
\hline $14-16$ años & 45 & 24,6 & 2 & 1,1 & 47 & 12,8 & $X^{2}=157,669$ \\
\hline $17-19$ años & 101 & 55,2 & 26 & 14,2 & 127 & 34,7 & P-valor $=0,000$ \\
\hline $20-24$ años & 35 & 19,1 & 132 & 72,1 & 167 & 45,6 & $\mathrm{OR}=29,51$ \\
\hline $25-29$ años & 2 & 1,1 & 21 & 11,5 & 23 & 6,3 & $\begin{array}{c}\text { IC95 } \%: 7,04- \\
123,76\end{array}$ \\
\hline 30 a más años & 0 & 0,0 & 2 & 1,1 & 2 & 0,5 & $\begin{array}{l}\text { Exp.:14 y } 16 \\
\begin{array}{c}\text { No Exp:> } 17 \\
\text { años }\end{array}\end{array}$ \\
\hline Total & 183 & 100,0 & 183 & 100,0 & 366 & 100,0 & \\
\hline \multicolumn{8}{|c|}{ Número de parejas sexuales } \\
\hline Única & 50 & 27,3 & 157 & 85,8 & 207 & 56,6 & $X^{2}=127,315$ \\
\hline 2 a más parejas & 133 & 72,7 & 26 & 14,2 & 159 & 43,4 & $\begin{array}{l}\text { P-valor }=0,000 \\
\text { OR }=16,06\end{array}$ \\
\hline Total & 183 & 100,0 & 183 & 100,0 & 366 & 100,0 & $\begin{array}{c}\text { IC95\%:9,48- } \\
27,21 \\
\text { Exp.: + de dos } \\
\text { parejas } \\
\text { No Exp: Pareja } \\
\text { única } \\
\end{array}$ \\
\hline \multicolumn{8}{|c|}{ ITS (últimos 5 años) } \\
\hline No & 74 & $\begin{array}{l}40,4 \\
59,6\end{array}$ & 144 & $\begin{array}{l}78,7 \\
21,3\end{array}$ & 218 & $\begin{array}{l}59,6 \\
40,4\end{array}$ & $\begin{array}{c}\mathrm{X}^{2}=55,585 \\
\text { P-valor }=0,000 \\
\mathrm{OR}=5,44\end{array}$ \\
\hline Total & 183 & 100,0 & 183 & 100,0 & 366 & 100,0 & $\begin{array}{c}\text { IC95\%:3,43- } \\
8,62 \\
\text { Exp.: Presencia } \\
\text { ITS } \\
\text { No Exp: No } \\
\text { ITS. }\end{array}$ \\
\hline
\end{tabular}




\begin{tabular}{cccccccc}
\hline $\begin{array}{c}\text { Tipo de ITS } \\
\text { VPH }\end{array}$ & 64 & 58,7 & 14 & 35,9 & 78 & 52,7 & $\mathrm{X}^{2}=9,108$ \\
Sífilis & 1 & 0,9 & 0 & 0,0 & 1 & 0,7 & P-valor=0,058 \\
SIDA & 0 & 0,0 & 0 & 0,0 & 0 & 0,0 & OR=2,54 \\
$\begin{array}{c}\text { Candidiasis/ } \\
\text { Trichomona } \\
\text { Condiloma }\end{array}$ & 21 & 19,3 & 16 & 41,0 & 37 & 25,0 & \\
acuminado & 1 & 0,9 & 0 & 0,0 & 1 & 0,7 & \\
$\begin{array}{c}\text { Herpes genital } \\
\text { Trichomonas }\end{array}$ & 0 & 0,0 & 0 & 0,0 & 0 & 0,0 & \\
\hline Total & 22 & 20,2 & 9 & 23,1 & 31 & 20,9 & \\
\hline
\end{tabular}

En el análisis estadístico se encontró una asociación significativa de los factores de riesgo sexual como, el inicio de las relaciones sexuales entre los 14-16 años, con 2 o más compañeros sexuales, presencia de ITS en los últimos 5 años, y dentro de los tipos de ITS, el VPH con las lesiones precancerosas del cuello uterino con un $\mathrm{OR}=29,51$; $\mathrm{OR}=16,06 ; \mathrm{OR}=5,54$ y $\mathrm{OR}=2,54$ respectivamente; expresa este mismo riesgo a nivel poblacional lo que se traduce en un intervalo de confianza al 95\% >1 y expresa significancia de estos riesgos al verificar que la influencia del azar, es decir, el valor de p es inferior a 5\%; se concluye que el inicio de las relaciones sexuales entre los 14-16 años, contacto sexual con 2 o más compañeros sexuales, presencia de ITS en los últimos 5 años y el VPH, se constituyeron factores de riesgo para desarrollar lesiones precancerosas de cuello uterino, el resto de los factores no mostraron asociación significativa con las lesiones precancerosas de cuello uterino.

Tabla 3. Factores reproductivos asociados a lesiones precancerosas del cuello uterino

\begin{tabular}{|c|c|c|c|c|c|c|c|}
\hline \multirow{2}{*}{$\begin{array}{c}\text { Factores } \\
\text { reproductivos }\end{array}$} & \multicolumn{2}{|c|}{$\begin{array}{l}\text { Con lesiones y/o } \\
\text { cáncer cervical }\end{array}$} & \multicolumn{2}{|c|}{$\begin{array}{l}\text { Sin lesiones ni } \\
\text { cáncer cervical }\end{array}$} & \multicolumn{2}{|c|}{ Total } & \multirow{2}{*}{$\begin{array}{l}\text { Resultados aná- } \\
\text { lisis estadístico }\end{array}$} \\
\hline & $\mathrm{n}$ & $\%$ & $\mathrm{n}$ & $\%$ & $\mathrm{n}$ & $\%$ & \\
\hline \multicolumn{8}{|l|}{ Gestaciones } \\
\hline Nuligesta & 3 & 1,6 & 36 & 19,7 & 39 & 10,7 & $X^{2}=152,653$ \\
\hline Primigesta & 33 & 18,0 & 117 & 63,9 & 150 & 41,0 & $P$-valor $=0,000$ \\
\hline Multigesta & 130 & 71,0 & 28 & 15,3 & 158 & 43,2 & $\mathrm{OR}=20,83$ \\
\hline Gran multigesta & 17 & 9,3 & 2 & 1,1 & 19 & 5,2 & $\begin{array}{c}\text { IC } 95 \%: 12,20- \\
35,55\end{array}$ \\
\hline
\end{tabular}


Exp.: Multi/

gran mul

Total

100,0

183

100,0

366100,0

No Exp: Nulig y

primig.

\section{Paridad}

\begin{tabular}{|c|c|c|c|c|c|c|c|}
\hline Nulípara & 3 & 1,6 & 36 & 19,7 & 39 & 10,7 & $X^{2}=138,245$ \\
\hline Un parto & 43 & 23,5 & 120 & 65,6 & 163 & 44,5 & P-valor $=0,000$ \\
\hline $\begin{array}{c}\text { Dos a cinco } \\
\text { partos }\end{array}$ & 122 & 66,7 & 25 & 13,7 & 147 & 40,2 & $\mathrm{OR}=17,21$ \\
\hline Más de 5 partos & 15 & 8,2 & 2 & 1,1 & 17 & 4,6 & $\begin{array}{c}\text { IC95\%:10,15- } \\
29,17\end{array}$ \\
\hline Total & 183 & 100,0 & 183 & 100,0 & 366 & 100,0 & $\begin{array}{c}\text { Exp.: Multip/ } \\
\text { gran } \\
\text { No Exp: Nulip/ } \\
\text { prim. }\end{array}$ \\
\hline \multicolumn{8}{|c|}{ Uso de método anticonceptivo (MAC) } \\
\hline Hormonal & 110 & 60,1 & 86 & 47,0 & 196 & 53,6 & $X^{2}=6 ., 27$ \\
\hline No hormonal & 73 & 39,9 & 97 & 53,0 & 170 & 46,4 & P-valor $=0,012$ \\
\hline Total & 183 & 100,0 & 183 & 100,0 & 366 & 100,0 & $\begin{array}{c}\text { OR=1,70 } \\
\text { IC95\%:1,12- } \\
2,57 \\
\text { Exp. Hormonal. } \\
\text { No Exp.: No } \\
\text { hormonal }\end{array}$ \\
\hline \multicolumn{8}{|c|}{ Tipo de MAC hormonal } \\
\hline Inyectable & 58 & 52,7 & 66 & 76,7 & 124 & 63,3 & $X^{2}=1, .979$ \\
\hline Píldoras & 52 & 47,3 & 20 & 23,3 & 72 & 36,7 & P-valor $=0,001$ \\
\hline Total & 110 & 100,0 & 86 & 100,0 & 196 & 100,0 & \\
\hline \multicolumn{8}{|c|}{ Tiempo de uso MAC } \\
\hline Menos de 1 año & 16 & 14,5 & 6 & 7,0 & 22 & 11,2 & $X^{2}=12,357$ \\
\hline Entre 1 a 2 años & 46 & 41,8 & 54 & 62,8 & 100 & 51,0 & P-valor $=0,006$ \\
\hline Entre 3 a 4 años & 26 & 23,6 & 20 & 23,3 & 46 & 23,5 & \\
\hline Más de 5 años & 22 & 20,0 & 6 & 7,0 & 28 & 14,3 & \\
\hline Total & 110 & 100,0 & 86 & 100,0 & 196 & 100,0 & \\
\hline
\end{tabular}




\begin{tabular}{cccccccc}
\hline $\begin{array}{c}\text { Resultados del } \\
\text { PAP }\end{array}$ & & & & & & & \\
Ninguno & 158 & 86,3 & 0 & 0,0 & 158 & 86,3 & $\mathrm{X}^{2}=369,022$ \\
$\begin{array}{c}\text { Inflamación Leve } \\
\text { Inflamación } \\
\text { moderada }\end{array}$ & 1 & 0,5 & 0 & 0,0 & 1 & 0,5 & P-valor $=0,000$ \\
$\begin{array}{c}\text { Inflamación } \\
\text { Severa }\end{array}$ & 13 & 7,1 & 0 & 0,0 & 13 & 7,1 & \\
\hline Total & 11 & 6,0 & 0 & 0,0 & 11 & 6,0 & \\
\hline
\end{tabular}

Evidenciándose luego del análisis estadístico, una asociación muy significativa entre los factores de riesgo reproductivo: Multigestación y multiparidad con las lesiones precancerosas del cuello uterino, con un $\mathrm{OR}=20,83$; $\mathrm{OR}=17,21$ respectivamente, a un intervalo de confianza que no contiene la unidad y un $\mathrm{p}<0,05$; se concluye: La multigestación y la multiparidad se constituyeron en factores de riesgo para desarrollar lesiones precancerosas del cuello uterino.

Respecto a los MAC, realizando la prueba estadística de dependencia chi $^{2}$, se encontró una asociación entre uso del MAC hormonal y las lesiones precancerosas del cuello uterino a una confianza de $95 \%$, de los cuales el tipo inyectable es de mayor uso $(52,7 \%)$ y el tiempo de uso más frecuente es entre 1 y 2 años con un $(41,8 \%)$.

Tabla 4. Lesiones precancerosas del cuello uterino

\begin{tabular}{|c|c|c|c|c|c|c|}
\hline \multirow[t]{2}{*}{ Resultados del PAP } & \multicolumn{2}{|c|}{$\begin{array}{l}\text { Con lesiones y/o } \\
\text { Cáncer cervical }\end{array}$} & \multicolumn{2}{|c|}{$\begin{array}{l}\text { Sin lesiones / ni } \\
\text { Cáncer cervical }\end{array}$} & \multicolumn{2}{|c|}{ Total } \\
\hline & $\mathrm{n}$ & $\%$ & $\mathrm{n}$ & $\%$ & $\mathrm{n}$ & $\%$ \\
\hline LEIA+Carcinoma en situ & 11 & 6,0 & 0 & 0,0 & 11 & 6,0 \\
\hline LEIA+NIC III & 5 & 2,7 & 0 & 0,0 & 5 & 2,7 \\
\hline LEIA+NIC II & 17 & 9,3 & 0 & 0,0 & 17 & 9,3 \\
\hline $\begin{array}{l}\text { LEIA+Carcinoma en situ+NIC } \\
\text { II }\end{array}$ & 3 & 1,6 & 0 & 0,0 & 3 & 1,6 \\
\hline $\mathrm{LEIB}+\mathrm{PVH}$ & 1 & 0,5 & 0 & 0,0 & 1 & 0,5 \\
\hline LEIB+NIC I & 44 & 24,0 & 0 & 0,0 & 44 & 24,0 \\
\hline $\mathrm{LEIB}+\mathrm{NIC} \mathrm{I}+\mathrm{PVH}$ & 57 & 31,1 & 0 & 0,0 & 57 & 31,1 \\
\hline ASCUS-AGUS & 45 & 24,6 & 0 & 0,0 & 45 & 24,6 \\
\hline Total & 183 & 100,0 & 0 & 0,0 & 183 & 100,0 \\
\hline
\end{tabular}


La tabla 4 responde al objetivo 4, se evidencia que el 31,1\% de las mujeres con lesiones y/o cáncer cervical tienen resultados de Lesión Intraepitelial Escamosa de bajo grado (LEIB) + Neoplasia Intraepitelial Cervical I (NIC I) + VPH; seguido de un 24,0\% de las mujeres con lesión que tienen LEIB+NIC I.

\section{DISCUSIÓN}

Los resultados de la investigación, respecto al primer objetivo, orientado a identificar los factores de riesgo personal para desarrollar lesiones precancerosas del cuello uterino, se dio a conocer en la tabla 1, la cual nos demuestra que la procedencia del área rural y antecedentes familiares les corresponde riesgos muéstrales de 3,92 y 3,13 respectivamente; estas asociaciones muestrales fueron verificadas a través de la prueba $\mathrm{chi}^{2}$, siendo el valor alcanzado suficiente para poder afirmar que la misma tiene significancia estadística $(\mathrm{p}<0,05)$ de tal forma se atribuye a dichas variables la condición de factor de riesgo para lesiones precancerosas, además la edad mayores de 35 años, el grado de instrucción primaria o ninguna les corresponde riesgos muestrales de 1,94 y 1,84 también constituyeron en factores de riesgo para desarrollar lesiones precancerosas del cuello uterino, habiéndose encontrado resultados opuesto en otras investigaciones tal es así, en Guatemala, Vicente (2014) plantea que no se encontró asociación como factor de riesgo a la edad, pero los que tienen mayor riesgo de presentar lesiones pre malignas del cérvix, son las mujeres del área urbana. Conclusión a la que también arribó Cevallos (2012) en Ecuador, las edades están comprendidas entre los 34 a 44 años, se relacionan con la presencia de lesiones en el cuello uterino. Por otro lado, lo hallado por May (2015) en México, la edad entre 35 a 39 años se asoció con algún tipo de Lesión Intraepitelial, los antecedentes heredofamiliares de CACU, fue estadísticamente significativa, además, Arango (2014) halló un mayor número de alteraciones citológicas en el grupo de 25 a 34 años. Entre tanto, Ramos (2015) concluyó, el 66,7\% de las pacientes con lesiones precancerosas del cuello uterino proceden de la zona rural y son analfabetas.

Las tablas 2 y 3 responden al segundo y tercer objetivo lo cual está orientado a reconocer si los factores de riesgo sexuales y reproductivos están asociadas a lesiones precancerosas de cuello uterino, a una confianza de 95\%. En relación a los factores de riesgo sexual, como el inicio de las relaciones sexuales entre los 14-16 años, mujeres que tuvieron más de dos compañeros sexuales, presencia de ITS en los últimos 5 años, y el VPH, les corresponde riesgos muéstrales de 29,51; 16,06; 5,54 y 2,54 respectivamente, estas asociaciones muestrales fueron verificadas a través de la prueba chi $^{2}$, siendo el valor alcanzado suficiente para poder afirmar que la misma tiene significancia estadística $(\mathrm{p}<0,05)$ de tal forma podremos atribuir a dichas variables la condición de factores de riesgo para las lesiones precancerosas del cuello uterino; además la edad de la menarquia también se considera como factor de riesgo no significativo para las lesiones precancerosas. Dentro de los antecedentes encontrados tenemos el estudio 
de Marruffo (2015) en Lima-Perú, identificó los factores de riesgo más reconocidos para lesiones del cuello uterino que fueron la presencia del VPH, inicio temprano de relaciones sexuales y tener muchos compañeros sexuales. Se precisa las conclusiones a las que llegó Castro (2014) en Sullana-Perú, el inicio de relaciones sexuales antes de los 18 años, más de dos parejas sexuales se asocian con las lesiones precancerosas de cuello uterino, así mis mo, Conde (2014) plantea el inicio temprano de las relaciones sexuales entre los 14 a 16 años, tener más de dos parejas sexuales, antecedentes de ITS, son factores significativos de riesgo asociados a lesiones precancerosas del cuello uterino. Al respecto May (2015) confirmó la asociación entre los antecedentes de VPH y el riesgo de lesiones intraepiteliales cervicales.

Los factores reproductivos: Multigestación y multiparidad se constituyeron en factores de riesgo para desarrollar lesiones precancerosas del cuello uterino. Con respecto al uso del MAC hormonal por más de cinco años y el tipo de MAC hormonal: El inyectable, se encontró que sí existe asociación con las lesiones precancerosas del cuello uterino. Al respecto Cordero (2015), en Cuba, sostiene que existe asociación significativamente entre los antecedentes obstétricos de partos y abortos provocados, partos en la adolescencia, utilización de anticoncepción oral, hábito de fumar y la susceptibilidad genética y la citología cervical alterada como factores de riesgo para cáncer del cuello uterino, además menciona a las relaciones sexuales con la menstruación y contra natura, antecedentes de ITS. Al respecto, Sánchez (2014) sostiene que los factores de riesgo reproductivo y las lesiones escamosas epiteliales de bajo y alto grado, el 100\% están relacionados significativamente con cada una de las displasias. Vicente (2014), en Guatemala, plantea que las mujeres que tienen mayor riesgo de presentar lesiones precancerosas del cuello uterino son las del área urbana, con vida sexual antes de los 20 años, con más de 5 hijos y 2 o más parejas sexuales. Así mismo, Campos (2015) concluyó, la paridad, el antecedente de haber abortado y el inicio de relaciones sexuales son factores de riesgo para lesiones precancerosas.

Así mismo, Conde (2014) encontró, el tener dos a cinco partos, uso de MAC hormonales representan factores significativos de riesgo. De igual manera, Ramos (2015) manifestó que las pacientes multíparas presentaban la mayor proporción de lesiones precancerosa con un $\mathrm{OR}=7,892$.

En la tabla 4 se observan las lesiones precancerosas del cuello uterino más frecuentes, que representan el 31,1\%, son aquellos con resultados: la Lesión Escamosa Intraepitelial de Bajo Grado (LEIB)+Neoplasia Intraepitelial Escamosa I (NIC I) + Papiloma Virus Humano(PVH). Como también afirma Ramos (2015), la Lesión Escamosa Intraepitelial de Bajo Grado más (LEIB) más PVH presentan mayor frecuencia de 41,7\%, seguido de la Lesión Escamosa Intraepitelial de Alto Grado con 30,2\%. 


\section{CONCLUSIONES}

Los factores de riesgo personal como la procedencia rural y antecedentes familiares se constituyen factores de riesgo para lesiones precancerosas del cuello uterino.

Se halló una asociación significativa de riesgo de los factores sexuales: El inicio de las relaciones sexuales entre los 14-16 años, contacto sexual con 2 o más compañeros sexuales, presencia de ITS en los últimos 5 años y el VPH con las lesiones precancerosas del cuello uterino.

Se encontró asociación significativa de riesgo entre los factores reproductivos y lesiones precancerosas del cuello uterino: Multigestacion, multiparidad, el uso de MAC hormonal inyectable y antecedentes familiares de cáncer del cuello uterino.

El tipo de lesión precancerosa más frecuente del cuello uterino corresponde a la Lesión Escamosa Intraepitelial de Bajo Grado (LEIB)+ Neoplasia Intraepitelial Escamosa I (NIC I) + Papiloma Virus Humano (PVH).

\section{REFERENCIAS BIBLIOGRÁFICAS}

Arango, Gian. 2014. Prevalencia de lesiones premalignas de cáncer de cuello uterino en los resultados de Papanicolaou en las mujeres atendidas en el Hospital II-Cañete. Lima: Universidad Ricardo Palma.

Bonita, Ruth. 2008. Epidemiología básica. 2da. ed. Washington: OPS.

Castro, Balarezo. 2014. Edad de inicio de relaciones coitales y número de compañeros sexuales como factor de riesgo para el cáncer de cuello uterino en mujeres atendidas en el Hospital de Apoyo Sullana II-2. Tesis de Licenciatura. Tumbes: Universidad Privada Antenor Orrego.

Campos, Roberto. 2015. Papanicolaou anormal y factores de riesgo en pacientes con lesiones escamosas intraepiteliales Hospital Carlos Lanfranco La Hoz, en el período de enero 2009 a diciembre 2013. Lima: Universidad San Martín de Porres.

Cevallos, Boada. 2012. Hallazgos anormales de citología cervical y factores relacionados con la historia reproductiva de la mujer en usuarias atendidas en Solca Núcleo Ibarra periodo enero a junio 2012. Tesis Doctorado. Quito: Universidad Técnica del Norte.

Conde, Alicia. 2014. Factores de riesgo, asociados a lesiones en el cuello uterino, en mujeres en edad fértil Hospital María Auxiliadora julio- agosto 2013. Tesis de Licenciatura. Lima: Universidad San Martín de Porres.

Cordero, Martínez. 2015. Citologías alteradas y algunos factores de riesgo para el cáncer cervicouterino, en el Hospital General Docente "Leopoldito Martínez" 2011-2012-Cuba. 
May, Ruth. 2015. Factores de riesgo asociados a las lesiones intraepiteliales, cervicales, Balancán. Tabasco 2010. Revista Salud en Tabasco Vol. 21, No. 2 y 3, p. 62-70. <http://salud.tabasco.gob.mx/content/revista >. [Consulta: 26-03-2016].

Marruffo, Betty. 2015. Análisis de los factores de riesgo para cáncer de cuello uterino en pacientes atendidas en el consultorio de ginecología del Hospital Central PNP Luis N. Sáenz 2015. Tesis de Licenciatura. Lima: Universidad Ricardo Palma.

OPS. 2017. 29.a conferencia sanitaria panamericana 69.a sesión del comité regional de la OPS para las américas Washington, D.C., EUA, del 25 al 29 de septiembre del 2017. <file:// C:/Users/usuario/Downloads/CSP29-INF-4-s.pdf.). [Consulta: 26-03-2016].

Ramos, Dávila. 2015. Factores de riesgo relacionados a lesiones pre malignas de cáncer del cuello uterino. Hospital Víctor Ramos Guardia. Huaraz 2008-2012. Tesis de Licenciatura. Trujillo: Universidad Privada Antenor Orrego.

Sánchez, Elcira. 2014. Factores de riesgo reproductivo de mujeres con citología cervical uterina alterada, atendidas en el preventorio del Hospital General Jaén, enero-diciembre del 2014. Tesis de Licenciatura. Lima: Universidad Alas Peruanas.

Vicente, Edgar. 2014. Factores de riesgo epidemiológico asociados a lesiones premalignas de cérvix en pacientes con Papanicolaou en el Hospital Nacional de Occidente 2011. Tesis Maestría. Universidad de Guatemala.

Recepción: 30/04/2018

Aceptación: 30/05/2018

\section{Correspondencia}

Maximiliana Quispe Gómez

mquispes@hotmail.com 